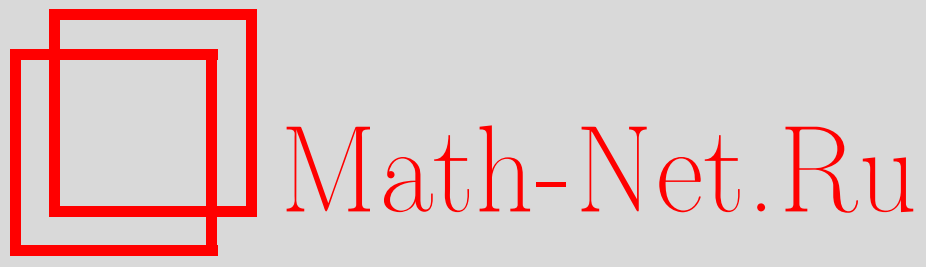

Ш. А. Аюпов, Н. А. Азамов, Представление косоэрмитовых элементов в алгебрах фон Неймана косыми коммутаторами, Функи.. анализ и его прил., 2001, том 35, выпуск 3, 75-77

DOI: https://doi.org/10.4213/faa261

Использование Общероссийского математического портала MathNet.Ru подразумевает, что вы прочитали и согласны с пользовательским соглашением

http://www . mathnet.ru/rus/agreement

Параметры загрузки:

IP: 3.95 .254 .165

26 апреля 2023 г., 12:18:56

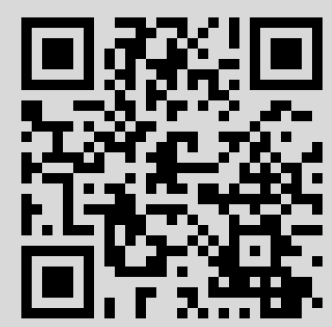


- $\mathscr{H}(t))$, где $\mathscr{H}(t)$ определена формулой $(4)$, является в силу теоремы 3 условно $\left(\mathbb{R}^{2}, \mathbb{R}^{2}\right)$-приводимой, причем оператор-функция, осуществляющая это подобие, может быть явно выписана. Отметим, что для $\widetilde{\mathscr{H}}(t)$ не выполняются условия приводимости к блочно-диагональной форме из $[1,2]$.

\title{
ЛИТЕРАТУРА
}

1. Курина Г. А., Мартыненко Г. В. Матем. заметки, 66, вып. 5, 688-695 (1999). 2. Курина Г. А., Мартыненко Г. В. Докл. РАН, 371, № 5, 594-596 (2000). 3. Азизов Т. Я., Иохвидов И. С. Основы теории линейных операторов в пространствах с индефинитной метрикой. Наука, М., 1986. 4. Далец̧кий Ю. Л., Крейн М. Г. Устойчивость решений дифференциальных уравнений в банаховом пространстве. Наука, M., 1970. 5. Sibuya Y. Math. Ann., 4, 67-77 (1965).

Воронедский государственный университет

Воронежский высший авиационный инженерный институт

Поступило в редакцию 29 августа 2000 г. Воронежская государственная лесотехническая академия

В переработанном виде 2 апреля 2001 г.

УДК 517.98

\section{Представление косоэрмитовых элементов в алгебрах фон Неймана косыми коммутаторами}

\author{
(C) 2001. Ш. А. Аюпов, Н. А. АзАмов
}

Имеется целый ряд работ, посвященных представлению элементов алгебры фон Неймана (особенно факторов) в виде коммутаторов (т.е. в виде $[x, y]=$ $x y-y x)$ или суммы коммутаторов. Обзор результатов и более подробную библиографию можно найти в работе [1]. Эта проблематика имеет важное значение как для более глубокого изучения самих алгебр фон Неймана, так и для исследования лиевой структуры в этих алгебрах $[1,2]$.

В настоящей заметке мы рассмотрим проблему представления косоэрмитовых элементов коммутаторами косоэрмитовых элементов (называемых косыми коммутаторами) в вещественных факторах.

Пусть $B(H)$ есть *-алгебра всех ограниченных линейных операторов в комплексном гильбертовом пространстве $H$. Напомним, что слабозамкнутая вещественная *-подалгебра $R$ в $B(H)$ называется вещественной алгеброй фон Неймана, если она содержит единичный оператор и $R \cap i R=\{0\}[2,3]$. Комплексная обертывающая алгебра фон Неймана для вещественной алгебры фон Неймана $R$ совпадает с ее комплексификацией $N=R+i R$. При этом отображение $\alpha: a+i b \mapsto a^{*}+i b^{*}$ из $N$ в $N$ является инволютивным (т.е. периода 2 ) *-антиавтоморфизмом алгебры $N$. Обратно, для любой алгебры фон Неймана $M$ и ее инволютивного *-антиавтоморфизма $\alpha$ множество $R=\left\{x \in M: \alpha(x)=x^{*}\right\}$ является вещественной алгеброй фон Неймана (подробнее см. [2-4]). Вещественная алгебра фон Неймана $R$ называется вещественным фактором, если ее центр $Z_{R}=\{x \in R: x y=y x$ для любого $y \in R\}$ совпадает с $\{\lambda \mathbf{1}: \lambda \in \mathbb{R}\}$, где $\mathbb{R}-$ поле вещественных чисел. 
Для вещественных факторов известны следующие результаты относительно представления косоэрмитовых элементов косыми коммутаторами.

1. Если $R-$ вещественный фактор типа $\mathrm{I}_{n}(3 \leqslant n \leqslant \infty)$, то всякий кососимметрический элемент из $R$ представим в виде одного косого коммутатора $[2,5]$.

2 . Если $R-$ собственно бесконечный вещественный фактор (т. е. фактор типа $\mathrm{II}_{\infty}$ или III), то всякий кососимметрический элемент есть сумма трех косых коммутаторов $[2,6]$.

3. Если $R$ - вещественный фактор типа $\mathrm{II}_{1}$, то всякий кососимметрический элемент есть сумма конечного числа косых коммутаторов $[2,6]$, причем вопрос об оценке количества слагаемых здесь открыт.

Представляется правдоподобным, что и в случаях 2 и 3 можно ограничиться одним косым коммутатором. В настоящей работе сделан шаг для доказательства этой гипотезы, а именно доказано, что всякий кососимметрический элемент вещественного фактора $R$ является косым коммутатором в обертывающей алгебре фон Неймана $N=R+i R$.

Рассмотрим комплексный или вещественный фактор $M$ в $B(H)$.

Лемма 1. Пусть $s$ u $t$-самосопряженные элементы фактора $M, a$

$$
s=\int \lambda d e_{\lambda} \quad u \quad t=\int \lambda d f_{\lambda}
$$

- спектральные разложения этих элементов. Если проекторы $e_{\lambda}-e_{\mu} u f_{\lambda}-f_{\mu}$ эквивалентны для любых $\lambda и \mu(\lambda>\mu)$, то существует унитарный элемент и из $M$, такой, что $u^{*} s u=t$.

Пусть $R$ - вещественный фактор, $N=R+i R-$ его комплексификация, $R_{k}$ и $N_{k}$ - кососимметрические части, а $R_{s}$ и $N_{s}$ - симметрические части факторов $R$ и $N$ соответственно. Пусть $k$ - кососимметрический элемент из $R, k=i s$, где $s \in N_{s}$, и $s=s_{+}-s_{-}-$разложение элемента $s$ в разность двух симметрических положительных дизъюнктных элементов.

Лемма 2. Если $R$ - вещественный фактор, то в факторе $N=R+i R$ существует унитарный элемент $u$, такой, ито $u s_{+} u^{*}=s_{-}$.

Носители $e_{+}$и $e_{-}$элементов $s_{+}$и $s_{-}$соответственно эквивалентны. Элемент $v=e_{-} u e_{+}$является частичной изометрией с начальным проектором $e_{+}$и конечным проектором $e_{-}$. Элементы $e_{+}, e_{-}$и $v$ образуют полный набор матричных элементов, так что всякий элемент из $N$ можно представить в виде $2 \times 2$-матрицы. Элементу $k$ в этом представлении соответствует матрица $\left(\begin{array}{cc}i s_{+} & 0 \\ 0 & -i s_{+}\end{array}\right)$. Автоморфизм $\beta(x)=u x u^{*}$, где $u=\frac{1}{\sqrt{2}}\left(\begin{array}{cc}1 & 1 \\ i & -i\end{array}\right) \otimes \mathbf{1}$, приводит $k$ к виду $\left(\begin{array}{cc}0 & s \\ -s & 0\end{array}\right)$, где $s-$ положительный элемент фактора $N$. Таким образом, установлена следующая

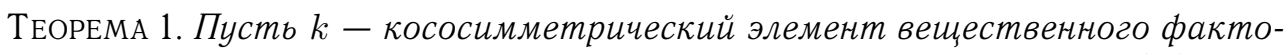
pa $R$. Существует изоморфизм фактора $R+i R$ на фактор вида $M_{2}(\mathbb{C}) \otimes M$, который переводит элемент $k$ в элемент $\left(\begin{array}{cc}0 & s \\ -s & 0\end{array}\right)$, где $s-$ положительный элемент фактора $M$. 
Теорему 1 можно рассматривать как обобщение на случай факторов известной теоремы из линейной алгебры о стандартном виде вещественной кососимметрической матрицы.

$$
\begin{aligned}
& \text { Пусть } s=\left(\begin{array}{cc}
s_{1} & 0 \\
0 & s_{2}
\end{array}\right) \text {. Тогда } \\
& k=\left(\begin{array}{ccccc}
0 & 0 & s_{1} & 0 \\
0 & 0 & 0 & s_{2} \\
-s_{1} & 0 & 0 & 0 \\
0 & -s_{2} & 0 & 0
\end{array}\right)=\left[\left(\begin{array}{cccc}
0 & 1 & 0 & 0 \\
-1 & 0 & 0 & 0 \\
0 & 0 & 0 & 0 \\
0 & 0 & 0 & 0
\end{array}\right),\left(\begin{array}{cccc}
0 & 0 & 0 & -s_{2} \\
0 & 0 & s_{1} & 0 \\
0 & -s_{1} & 0 & 0 \\
s_{2} & 0 & 0 & 0
\end{array}\right)\right] .
\end{aligned}
$$

Отсюда следует

ТЕОРЕмА 2. Всякий кососимметрический элемент вещественного фактора является коммутатором двух кососимметрических әлементов из обертывающцей алгебры фон Неймана.

СледствиЕ. Всякий кососимметрический элемент вещественного фактора $R$ можно представить в виде $\left[k_{1}, k_{2}\right]+\left[s_{1}, s_{2}\right]$, где $k_{1}, k_{2} \in R_{k}, s_{1}, s_{2} \in R_{s}$.

\title{
ЛИТЕРАТУРА
}

1. Fack Th., de la Harp P. Ann. Inst. Fourier, Grenoble, 30, No. 3, 49-70 (1980). 2. Ayupov Sh. A., Rakhimov A. A., Usmanov Sh. M. Jordan, Real and Lie Structures in Operator Algebras. Kluwer Acad. Publ., Dordrecht, 1997. 3. Størmer E. Pacif. J. Math., 21, No. 2, 349-370 (1967). 4. Аюпов Ш. А. Классификация и представление упорядоченных йордановых алгебр. Ташкент, Изд-во «ФАН», 1986. 5. Садовский В. Ю. Узб. матем. журн., No. 1, 55-63 (1994). 6. Ayuрол Sh. A., Azamov N. A. Comm. Algebra, 24, No. 4, 1501-1520 (1996).

Институт математики Академии Наук республики Узбекистан

Поступило в редакцию 22 июня 2000 г.

\section{К определению обобщенных многочленов Чебышёва над конечными полями}

\author{
(C) 2001. А. М. ВАшевНИК
}

1. Введение. Теория так называемых детских рисунков Гротендика, ведущая начало от работы [3], в настоящее время развивается довольно интенсивно. Часть этой теории связывает комбинаторно-топологические объекты (графы на поверхностях, которые и называются детскими рисунками) с алгебраическими «функциями Белого», определенными над полем комплексных чисел; см. [4].

Однако функции Белого имеют смысл и над произвольным полем и допускают независимое изучение.

В настоящей работе делается шаг в направлении распространения частного случая теории (в котором функции Белого сводятся к обобщенным многочленам Чебышёва) на произвольные алгебраически замкнутые поля. 